\title{
ON CERTAIN PAIRS OF AUTOMORPHISMS OF $C^{*}$-ALGEBRAS
}

\author{
C. J. K. BATTY \\ - (Received 8 April 1987) \\ Communicated by R. O. Vyborny
}

\begin{abstract}
Let $\alpha$ and $\beta$ be *-automorphisms of a $C^{*}$-algebra $A$ such that $\alpha+\alpha^{-1}=\beta+\beta^{-1}$. There exist invariant ideals $I_{1}, I_{2}$ and $I_{3}$ of $A$, with $I_{1} \cap I_{2} \cap I_{3}=\{0\}$, containing, respectively, the range of $\beta-\alpha$, the range of $\beta-\alpha^{-1}$, and the union of the ranges of $\beta^{2}-\alpha^{2}$ and $\beta^{2}-\alpha^{-2}$. The induced actions on the quotient algebras give a decomposition of the system $(A, \alpha, \beta)$ into systems where $\beta=\alpha, \beta=\alpha^{-1}$ and $\beta^{2}=\alpha^{2}=\alpha^{-2}$.

If $\alpha$ and $\beta$ are one-parameter groups of *-automorphisms such that $\alpha+\alpha^{-1}=\beta+\beta^{-1}$, then the corresponding result is valid, and may be strengthened to assert that $I_{1} \cap I_{2}=\{0\}$.

These results are analogues and extensions of similar results of A. B. Thaheem et al. for von Neumann algebras and commuting automorphisms.
\end{abstract}

1980 Mathematics subject classification (Amer. Math. Soc.) (1985 Revision): 46 L 40.

Keywords and phrases: automorphism, von Neumann algebra, $C^{*}$-algebra, central projection, ideal, derivation.

\section{Introduction}

Let $\alpha$ and $\beta$ be *-automorphisms of a $C^{*}$-algebra $A$. The equation

$$
\alpha+\alpha^{-1}=\beta+\beta^{-1}
$$

has been studied by Thaheem and Awami $[6,1,2]$. It was shown in [6] that if $A$ is a von Neumann algebra and $\alpha$ and $\beta$ commute, then there is a central projection $p$ in $A$ such that $\beta|A p=\alpha| A p$ and $\beta\left|A(1-p)=\alpha^{-1}\right| A(1-p)$. A short proof of a slightly stronger result (due to the author, and communicated

(C) 1989 Australian Mathematical Society $0263-6115 / 89 \$ A 2.00+0.00$ 
to Thaheem) was given in $[1,2]$. When $A$ is a $C^{*}$-algebra, the result for von Neumann algebras may be applied in $A^{* *}$ to deduce that, if $\alpha$ and $\beta$ commute, then there are orthogonal ideals $I_{1}$ and $I_{2}$, invariant under $\alpha, \alpha^{-1}, \beta$ and $\beta^{-1}$, such that $\beta(a)-\alpha(a) \in I_{1}$ and $\beta(a)-\alpha^{-1}(a) \in I_{2}$ for all $a$ in $A$. Considering the quotients $A / I_{1}$ and $A / I_{2}$, this may be regarded as a satisfactory description of $\beta$ being composed of $\alpha$ on one part of $A$ and $\alpha^{-1}$ on another.

The question arises as to the conclusions which may be drawn if $\alpha$ and $\beta$ are not assumed to commute. If $\alpha$ is inner, implemented by a unitary $u$, then it follows from (1) that

$$
2 u=\beta(u)+\beta^{-1}(u)
$$

Since $u$ is an extreme point of the unit ball of $A, \beta(u)=u$, so $\alpha$ and $\beta$ commute. In particular, if $A$ is a type I factor, then $\alpha$ and $\beta$ are automatically inner, and it follows that $\beta=\alpha$ or $\beta=\alpha^{-1}$ (since $p=0$ or $p=1$ ).

It is easily seen that (1) implies that $\alpha^{n}+\alpha^{-n}=\beta^{n}+\beta^{-n}$ for all integers $n$. On the other hand, it was shown in [7] that if $\alpha$ and $\beta$ are (weakly continuous) actions of $\mathbf{R}$ on a von Neumann algebra $A$ and

$$
\alpha_{t}+\alpha_{-t}=\beta_{t}+\beta_{-t} \text { for all } t
$$

then there is a central projection $p$, invariant under $\alpha$ and $\beta$, such that $\beta_{t} \mid A p=$ $\alpha_{t} \mid A p$ and $\beta_{t}\left|A(1-p)=\alpha_{-t}\right| A(1-p)$ for all $t$. This fact (in a case where it is already clear that $\alpha$ and $\beta$ commute) has been applied to the study of Tomita-Takesaki theory $[3,4]$.

When $\alpha$ and $\beta$ are (strongly continuous) actions of $\mathbf{R}$ on a $C^{*}$-algebra $A$ satisfying (2), it is not immediately possible to obtain a description of $\alpha$ and $\beta$ by passing to $A^{* *}$, because the extended actions may not be continuous. However, the proof in [7] (which uses spectral subspaces) can be modified to show that there are orthogonal ideals $I_{1}$ and $I_{2}$ in $A$, invariant under $\alpha$ and $\beta$, such that $\beta_{t}(a)-\alpha_{t}(a) \in I_{1}$ and $\beta_{t}(a)-\alpha_{-t}(a) \in I_{2}$ for all $a$ in $A$ and all real $t$.

In this paper, the equation (1) will be studied without assuming commutativity of $\alpha$ and $\beta$. For a $C^{*}$-algebra $A$, it will be shown in Section 3 that there are ideals $I_{1}, I_{2}$ and $I_{3}$ in $A$, invariant under $\alpha, \alpha^{-1}, \beta$ and $\beta^{-1}$, containing, respectively, the range of $\beta-\alpha$, the range of $\beta-\alpha^{-1}$, and the union of the ranges of $\beta^{2}-\alpha^{2}$ and $\beta^{2}-\alpha^{-2}$. The proof depends on an inspection of the weak closure of $A$ in its atomic representation, which is a direct sum of type I factors. Preliminary results for type I factors, giving necessary and sufficient conditions for (1) and similar equations, are given in Section 2 . In Section 4, it is shown how the same methods lead to proofs of the corresponding results for one-parameter groups of automorphisms. 


\section{Type I factors}

The most delicate case in the study of two automorphisms of a $C^{*}$-algebra $A$ will occur when they each interchange two of the direct summands of the weak closure of $A$ in its atomic representation. It is therefore necessary to study pairs of automorphisms of a direct sum of two type I factors. For this, it is convenient first to consider three or four automorphisms of a single type I factor.

Proposition 2.1. Let $\alpha, \beta$ and $\gamma$ be *-automorphisms of a type I factor $\mathscr{N}$, such that

$$
1+\alpha=\beta+\gamma, \quad 1+\alpha^{-1}=\beta^{-1}+\gamma^{-1} .
$$

Then at least one of the following conditions holds:

(4.1) $\beta=1, \gamma=\alpha$,

(4.2) $\beta=\alpha, \gamma=1$,

(4.3) There exist a projection $f$ in $\mathscr{M}$ and a scalar $\lambda$ in $\mathbf{T}=\{z \in \mathbb{C}:|z|=1\}$ such that

$$
\alpha=\operatorname{Ad}\left(f-f^{\prime}\right), \quad \beta=\operatorname{Ad}\left(f+\lambda f^{\prime}\right), \quad \gamma=\operatorname{Ad}\left(f-\lambda f^{\prime}\right),
$$

where $f^{\prime}=1-f$.

Proof. Suppose that $\alpha=\operatorname{Ad} u, \beta=\operatorname{Ad} v, \gamma=\operatorname{Ad} w$. Then

$$
x+u x u^{*}=v x v^{*}+w x w^{*} \quad(x \in \mathscr{K}) .
$$

Putting $x=u$,

$$
2 u=v u v^{*}+w u w^{*} .
$$

Since $u$ is an extreme point of the unit ball of $\mathscr{M}, u=v u v^{*}=w u w^{*}$, so $u v=v u$, $u w=w u$. Putting $x=v$,

$$
2 v=v+w v w^{*},
$$

so $v w=w v$. Thus $\alpha, \beta$ and $\gamma$ commute.

It follows from (3) that

$$
\begin{aligned}
\alpha+\alpha^{-1} & =(1+\alpha)\left(1+\alpha^{-1}\right)-2=(\beta+\gamma)\left(\beta^{-1}+\gamma^{-1}\right)-2 \\
& =\beta \gamma^{-1}+\gamma \beta^{-1} .
\end{aligned}
$$

Hence $\alpha=\beta \gamma^{-1}$ or $\alpha=\gamma \beta^{-1}$ (see the Introduction).

Suppose that $\alpha=\gamma \beta^{-1}$ (the other case is similar), so $\gamma=\alpha \beta$. Let $R$ be the range of $1-\beta$. If $R=\{0\}$, then $\beta=1$ and (4.1) holds.

Suppose that $R \neq\{0\}$. The algebra generated by $R$ is ultraweakly dense in $\mathscr{A}[6]$. Since

$$
(1+\alpha)(1-\beta)=1+\alpha-\beta-\alpha \beta=1+\alpha-\beta-\gamma=0,
$$


$\alpha(x)=-x$ for any $x$ in $R$, so $\alpha^{2}(x)=x$. The fixed-point set of $\alpha^{2}$ is an ultraweakly closed subalgebra of $\mathscr{M}$ containing $R$, and is therefore equal to $\mathscr{M}$, so $\alpha^{2}=1$. Multiplying $u$ by a scalar, it is therefore possible to assume that $u^{2}=1$, so $u=f-f^{\prime}$ for some projection $f$. Since $(1+\alpha)(1-\beta)=0$,

$$
0=f\left((1+\alpha)\left(x-v x v^{*}\right)\right) f=2 f\left(x-v x v^{*}\right) f \quad(x \in \mathscr{M}) .
$$

Since $f v=v f$, it follows that $f v=\lambda_{1} f$ for some $\lambda_{1}$ in T. Similarly $f^{\prime} v=\lambda_{2} f^{\prime}$ for some $\lambda_{2}$ in $\mathbf{T}$. Let $\lambda=\lambda_{2} / \lambda_{1}$, so $v=\lambda_{1}\left(f+\lambda f^{\prime}\right)$. Then

$$
\begin{aligned}
& \beta=\operatorname{Ad} v=\operatorname{Ad}\left(f+\lambda f^{\prime}\right), \\
& \gamma=\alpha \beta=\operatorname{Ad}\left(\left(f-f^{\prime}\right)\left(f+\lambda f^{\prime}\right)\right)=\operatorname{Ad}\left(f-\lambda f^{\prime}\right) .
\end{aligned}
$$

REMARKS 2.2. 1. Proposition 2.1 remains valid for any inner automorphisms $\alpha, \beta$ and $\gamma$ of any factor $\mathscr{M}$, satisfying (3). (Indeed, it is sufficient to assume that two of the three automorphisms are inner.)

2. It is easily checked that each of (4.1), (4.2) and (4.3) implies (3).

COROLLARY 2.3. Let $\alpha_{1}, \alpha_{2}, \beta_{1}$ and $\beta_{2}$ be *-isomorphisms of a type I factor $\mathscr{M}_{1}$ onto a type I factor $\mathscr{M}_{2}$, such that

$$
\alpha_{1}+\alpha_{2}=\beta_{1}+\beta_{2}, \quad \alpha_{1}^{-1}+\alpha_{2}^{-1}=\beta_{1}^{-1}+\beta_{2}^{-1} .
$$

Then at least one of the following conditions holds:

(6.1) $\beta_{1}=\alpha_{1}, \beta_{2}=\alpha_{2}$,

(6.2) $\beta_{1}=\alpha_{2}, \beta_{2}=\alpha_{1}$,

(6.3) There exist a projection $f$ in $\mathscr{M}$ and a scalar $\lambda$ in $\mathrm{T}$ such that

$\alpha_{2}=\alpha_{1} \circ \operatorname{Ad}\left(f-f^{\prime}\right), \quad \beta_{1}=\alpha_{1} \circ \operatorname{Ad}\left(f+\lambda f^{\prime}\right), \quad \beta_{2}=\alpha_{1} \circ \operatorname{Ad}\left(f-\lambda f^{\prime}\right)$, where $f^{\prime}=1-f$.

Proof. The automorphisms $\alpha=\alpha_{1}^{-1} \alpha_{2}, \beta=\alpha_{1}^{-1} \beta_{1}$ and $\gamma=\alpha_{1}^{-1} \beta_{2}$ satisfy (3), and an application of Proposition 2.1 gives the result immediately.

COROLLARY 2.4. Let $\mathscr{K}=\mathscr{M}_{1} \oplus \mathscr{M}_{2}$, where $\mathscr{M}_{1}$ and $\mathscr{M}_{2}$ are type I factors, and let $\alpha$ and $\beta$ be *-automorphisms of $\mathscr{M}$ such that $\alpha+\alpha^{-1}=\beta+\beta^{-1}$ and $\alpha\left(e_{1}\right)=\beta\left(e_{1}\right)=e_{2}$, where $e_{j}$ is the identity of $\mathscr{M}_{j}$. Then at least one of the following conditions holds:

(7.1) $\beta=\alpha$,

(7.2) $\beta=\alpha^{-1}$,

(7.3) There exist a $*$-isomorphism $\alpha_{1}: \mathscr{M}_{1} \rightarrow \mathscr{M}_{2}$, a projection $f$ in $\mathscr{M}_{1}$ and a scalar $\lambda$ in $\mathbf{T}$ such that

$$
\begin{aligned}
& \alpha(x \oplus y)=\left(f-f^{\prime}\right) \alpha_{1}^{-1}(y)\left(f-f^{\prime}\right) \oplus \alpha_{1}(x), \\
& \beta(x \oplus y)=\left(f-\bar{\lambda} f^{\prime}\right) \alpha_{1}^{-1}(y)\left(f-\lambda f^{\prime}\right) \oplus \alpha_{1}\left(\left(f+\lambda f^{\prime}\right) x\left(f+\bar{\lambda} f^{\prime}\right)\right),
\end{aligned}
$$

where $f^{\prime}=e_{1}-f$. 
Proof. There are *-isomorphisms $\alpha_{1}, \alpha_{2}, \beta_{1}$ and $\beta_{2}$ of $\mathscr{M}_{1}$ onto $\mathscr{M}_{2}$ such that

$$
\begin{aligned}
& \alpha(x \oplus y)=\alpha_{2}^{-1}(y) \oplus \alpha_{1}(x), \\
& \beta(x \oplus y)=\beta_{2}^{-1}(y) \oplus \beta_{1}(x) .
\end{aligned}
$$

Then $\alpha_{1}, \alpha_{2}, \beta_{1}$ and $\beta_{2}$ satisfy (5), so (6.1), (6.2) or (6.3) holds. These possibilities lead to (7.1), (7.2) or (7.3), respectively.

REMARK 2.5. If (7.3) holds, then $\beta^{2}(x \oplus y)=\alpha^{2}(x \oplus y)=\alpha^{-2}(x \oplus y)=\left(f-f^{\prime}\right) x\left(f-f^{\prime}\right) \oplus \alpha_{1}\left(f-f^{\prime}\right) y \alpha_{1}\left(f-f^{\prime}\right)$.

\section{Single automorphisms}

The following theorem gives conditions which are both necessary and sufficient for a pair of *-automorphisms to satisfy (1). Although the conditions are not entirely intrinsic to the $C^{*}$-algebra $A$ (they involve the weak closure of $A$ in a certain representation), they imply certain other conditions which are intrinsic, necessary, and close to being sufficient (see the subsequent corollaries).

THEOREM 3.1. Let $\alpha$ and $\beta$ be *-automorphisms of a $C^{*}$-algebra $A$ such that $\alpha+\alpha^{-1}=\beta+\beta^{-1}$. There exist (closed two-sided) ideals $I_{1}$ and $I_{2}$ of $A$, each invariant under $\alpha, \alpha^{-1}, \beta$ and $\beta^{-1}$, a representation $\pi$ of $A, *$-automorphisms $\bar{\alpha}$ and $\bar{\beta}$ of $\pi(A)^{\prime \prime}$, a projection $f$ in $\pi(A)^{\prime \prime}$, and a unitary $u$ in the centre $Z$ of $\pi(A)^{\prime \prime}$, such that

$$
\begin{aligned}
& \beta(a)-\alpha(a) \in I_{1}, \beta(a)-\alpha^{-1}(a) \in I_{2} \quad(a \in A), \\
& \pi \text { is faithful on } I_{1} \cap I_{2}, \\
& \bar{\alpha} \pi=\pi \alpha, \bar{\beta} \pi=\pi \beta \\
& \bar{\alpha}\left(f+u f^{\prime}\right)\left(f+u f^{\prime}\right) \in Z \\
& \bar{\alpha}^{2}=\operatorname{Ad}\left(f-f^{\prime}\right), \bar{\beta} \bar{\alpha}=\operatorname{Ad}\left(f+u f^{\prime}\right),
\end{aligned}
$$

where $f^{\prime}=1-f$.

Conversely, if there exist $I_{1}, I_{2}, \pi, \bar{\alpha}, \bar{\beta}, f$ and $u$ satisfying these conditions, then $\bar{\beta}^{2}=\operatorname{Ad}\left(f-f^{\prime}\right), \bar{\beta}^{-1} \bar{\alpha}=\operatorname{Ad}\left(f-u f^{\prime}\right)$, and $\alpha+\alpha^{-1}=\beta+\beta^{-1}$.

PROOF. Let $z$ be the atomic projection in $A^{* *}$, that is, the sum of the supports of the irreducible representations of $A$, and let $\mathscr{M}=z A^{* *}$, so that $\mathscr{M}$ is a direct sum of type I factors. Note that $z$ is invariant under the normal extensions of $\alpha$ and $\beta$. The induced automorphisms of $\mathscr{C}$ will also be denoted by $\alpha$ and $\beta$, so that (1) remains valid on $\mathscr{K}$. 
Let $\mathscr{E}$ be the set of all minimal central projections in $\mathscr{M}$, and

$$
\begin{aligned}
& \mathscr{E}_{1}=\{e \in \mathscr{E}: \beta|\mathscr{M} e=\alpha| \mathscr{K} e\}, \\
& \mathscr{E}_{2}=\left\{e \in \mathscr{E}: \beta\left|\mathscr{M} e=\alpha^{-1}\right| \mathscr{M} e\right\}, \\
& \mathscr{E}_{3}=\mathscr{E} \backslash\left(\mathscr{E}_{1} \cup \mathscr{E}_{2}\right) .
\end{aligned}
$$

For each $e$ in $\mathscr{E}$, it is claimed that

(8) $e \in \mathscr{E}_{j} \Rightarrow \alpha(e), \alpha^{-1}(e), \beta(e), \beta^{-1}(e) \in \mathscr{E}_{j}(j=1,2)$

(9) If $e \in \mathscr{E}_{3}$, and $\tilde{e}=\alpha(e)$, then $\alpha(\tilde{e})=e$ and there exist a projection $f_{e}$ in $\mathscr{M} e$ and a scalar $\lambda_{e}$ in $\mathbf{T}$ such that, for all $a$ in $A$,

(9.1) $\alpha^{2}(a) e=\left(f_{e}-f_{e}^{\prime}\right) a\left(f_{e}-f_{e}^{\prime}\right)$,

(9.2) $\beta \alpha(a) e=\left(f_{e}-\bar{\lambda}_{e} f_{e}^{\prime}\right) a\left(f_{e}-\lambda_{e} f_{e}^{\prime}\right)$,

(9.3) $\beta^{-1} \alpha(a) e=\left(f_{e}+\bar{\lambda}_{e} f_{e}^{\prime}\right) a\left(f_{e}+\lambda_{e} f_{e}^{\prime}\right)$, where $f_{e}^{\prime}=e-f_{e}$.

Since $\alpha(e)+\alpha^{-1}(e)=\beta(e)+\beta^{-1}(e)$, and all four terms are minimal central projections, there are four mutually exclusive cases:

1. $\alpha(e)=\beta(e) \neq \alpha^{-1}(e)=\beta^{-1}(e)$,

2. $\alpha(e)=\beta^{-1}(e) \neq \alpha^{-1}(e)=\beta(e)$,

3. $\alpha(e)=\alpha^{-1}(e)=\beta(e)=\beta^{-1}(e)=e$,

4. $\alpha(e)=\alpha^{-1}(e)=\beta(e)=\beta^{-1}(e) \neq e$.

Case 1. For $x$ in $\mathscr{H} e$,

$$
\alpha(x)+\alpha^{-1}(x)=\beta(x)+\beta^{-1}(x) .
$$

On each side, the two terms lie in different summands, so $\alpha(x)=\beta(x)$. Thus $e \in \mathscr{E}_{1} \backslash \mathscr{E}_{2}$. Since

$$
\alpha^{2}(x)+x=\beta \alpha(x)+\beta^{-1} \alpha(x),
$$

and the two terms on each side lie in different summands, it must be that $\beta \alpha(x)=$ $\alpha^{2}(x)$. Thus $\alpha(e) \in \mathscr{E}_{1}$. Since $\beta \alpha(e) \neq e, \alpha(e) \notin \mathscr{E}_{2}$. Similarly, $\alpha^{-1}(e) \in \mathscr{E}_{1} \backslash \mathscr{E}_{2}$.

Case 2. This is very similar to Case $1-$ now $e, \alpha(e)$ and $\alpha^{-1}(e)$ belong to $\mathscr{E}_{2} \backslash \mathscr{E}_{1}$

Case 3. In this case, $\alpha \mid \mathscr{K} e$ and $\beta \mid \mathscr{M} e$ are *-automorphisms of the type I factor $\mathscr{M} e$, satisfying (1), so $e \in \mathscr{E}_{1} \cup \mathscr{E}_{2}$ (see the Introduction). In this case, (8) is trivial.

Case 4. In this case, let $\tilde{e}=\alpha(e)$, so that $\mathscr{K}(e+\tilde{e})$ is a direct sum of two type I factors and is invariant under $\alpha, \alpha^{-1}, \beta$ and $\beta^{-1}$ which all interchange the two summands. Thus the restrictions of $\alpha$ and $\beta$ satisfy the conditions of Corollary 2.4, so at least one of (7.1), (7.2) and (7.3) holds on $\mathscr{M}(e+\tilde{e})$.

If (7.1) holds, then $e$ and $\tilde{e}$ belong to $\mathscr{E}_{1}$, and

$$
e \in \mathscr{E}_{2} \Leftrightarrow \alpha^{2} \mid \mathscr{M} e=1 \Leftrightarrow \tilde{e} \in \mathscr{E}_{2} .
$$


If (7.2) holds, then $e$ and $\tilde{e}$ belong to $\mathscr{E}_{2}$, and

$$
e \in \mathscr{E}_{1} \Leftrightarrow \alpha^{2} \mid \mathscr{K} e=1 \Leftrightarrow \tilde{e} \in \mathscr{E}_{1} .
$$

Finally, suppose that (7.3) holds, so that there exist a *-isomorphism $\alpha_{e}$ : $\mathscr{M} e \rightarrow \mathscr{M} \tilde{e}$, a projection $f_{e}$ in $\mathscr{M} e$ and a scalar $\lambda_{e}$ in $\mathbf{T}$ such that

(10.1) $\alpha(a) e=\left(f_{e}-f_{e}^{\prime}\right) \alpha_{e}^{-1}(a \tilde{e})\left(f_{e}-f_{e}^{\prime}\right)$,

(10.2) $\alpha(a) \tilde{e}=\alpha_{e}(a e)$

(10.3) $\beta(a) e=\left(f_{e}-\bar{\lambda}_{e} f_{e}^{\prime}\right) \alpha_{e}^{-1}(a \tilde{e})\left(f_{e}-\lambda_{e} f_{e}^{\prime}\right)$,

(10.4) $\beta(a) \tilde{e}=\alpha_{e}\left[\left(f_{e}+\lambda_{e} f_{e}^{\prime}\right) a\left(f_{e}+\bar{\lambda}_{e} f_{e}^{\prime}\right)\right]$.

Replacing $a$ by $\alpha(a)$ in (10.1) and (10.3), and using (10.2), gives (9.1) and (9.2). Replacing $a$ by $\beta^{-1} \alpha(a)$ in (10.4) and rearranging, using (10.2), gives (9.3). Thus (9) is satisfied. Furthermore,

$$
\begin{gathered}
e \in \mathscr{E}_{1} \Leftrightarrow f_{e}=0, f_{e}=e \text { or } \lambda_{e}=1 \Leftrightarrow \tilde{e} \in \mathscr{E}_{1}, \\
e \in \mathscr{E}_{2} \Leftrightarrow f_{e}=0, f_{e}=e \text { or } \lambda_{e}=-1 \Leftrightarrow e \in \mathscr{E}_{2} .
\end{gathered}
$$

Thus (8) is satisfied in this case.

Let

$$
I_{j}=\left\{a \in A: a e=0 \text { for all } e \text { in } \mathscr{E}_{j}\right\} \quad(j=1,2) .
$$

Then $I_{j}$ is an ideal, which is invariant under $\alpha, \alpha^{-1}, \beta$ and $\beta^{-1}$, by (8). For any $a$ in $A$ and $e$ in $\mathscr{E}_{1}$,

$$
\begin{gathered}
\beta^{-1}(e)=\alpha^{-1}(e), \\
(\beta(a)-\alpha(a)) e=\beta\left(a \beta^{-1}(e)\right)-\alpha\left(a \alpha^{-1}(e)\right)=0 .
\end{gathered}
$$

Thus $\beta(a)-\alpha(a) \in I_{1}$. Similarly, $\beta(a)-\alpha^{-1}(a) \in I_{2}$.

For each $e$ in $\mathscr{E}_{3}$, let $\left(\mathscr{H}_{e}, \pi_{e}\right)$ be an irreducible representation of $A$ with central support $e$. Let $(\mathscr{H}, \pi)=\bigoplus_{e \in \mathscr{E}_{3}}\left(\mathscr{H}_{e}, \pi_{e}\right)$. If $\pi(a)=0$ for some $a$ in $I_{1} \cap I_{2}$, then $a e=0$ for all $e$ in $\mathscr{E}$, so $a$ is annihilated by all irreducible representations of $A$. Thus $a=0$.

Since the representations $\pi_{e}$ are disjoint, $\pi(A)^{\prime \prime}$ may be identified with $\bigoplus_{e \in \mathscr{E}_{3}} \mathscr{M} e$ in such a way that $\pi(a)=\bigoplus a e$. Since $\mathscr{E}_{3}$ is invariant, $\alpha$ and $\beta$ induce *-automorphisms $\bar{\alpha}$ and $\bar{\beta}$ of $\pi(A)^{\prime \prime}$ satisfying $\bar{\alpha} \pi=\pi \alpha, \bar{\beta} \pi=\pi \beta$. Let $f=\bigoplus f_{e}, u=\bigoplus\left(-\bar{\lambda}_{e} e\right)$. Then $f$ is a projection and $u$ is a central unitary in $\pi(A)^{\prime \prime}$, and it follows from (9.1) that

$$
\bar{\alpha}^{2}(\pi(a))=\bigoplus \alpha^{2}(a) e=\bigoplus\left(f_{e}-f_{e}^{\prime}\right) a\left(f_{e}-f_{e}^{\prime}\right)=\left(f-f^{\prime}\right) \pi(a)\left(f-f^{\prime}\right),
$$

so $\bar{\alpha}^{2}=\operatorname{Ad}\left(f-f^{\prime}\right)$. Similarly, it follows from (9.2) that $\bar{\beta} \bar{\alpha}=\operatorname{Ad}\left(f+u f^{\prime}\right)$, and from (9.2) that $\bar{\beta}^{-1} \bar{\alpha}=\operatorname{Ad}\left(f-u f^{\prime}\right)$. Hence

$$
\begin{aligned}
\operatorname{Ad}\left(\bar{\alpha}\left(f+u f^{\prime}\right)\left(f+u f^{\prime}\right)\right) & =\bar{\alpha} \operatorname{Ad}\left(f+u f^{\prime}\right) \bar{\alpha}^{-1} \operatorname{Ad}\left(f-u f^{\prime}\right) \operatorname{Ad}\left(f-f^{\prime}\right)^{-1} \\
& =\bar{\alpha}(\bar{\beta} \bar{\alpha}) \bar{\alpha}^{-1}\left(\bar{\beta}^{-1} \bar{\alpha}\right) \bar{\alpha}^{-2} \\
& =1 .
\end{aligned}
$$

Thus $\bar{\alpha}\left(f+u f^{\prime}\right)\left(f+u f^{\prime}\right) \in Z$. 
Conversely, suppose that there exist $I_{1}, I_{2}, \pi, \bar{\alpha}, \bar{\beta}, f$ and $u$ as given. Then

$$
1=\operatorname{Ad}\left(\bar{\alpha}\left(f+u f^{\prime}\right)\left(f+u f^{\prime}\right)\right)=\bar{\alpha}(\bar{\beta} \bar{\alpha}) \bar{\alpha}^{-1}(\bar{\beta} \bar{\alpha})=\bar{\alpha} \bar{\beta}^{2} \bar{\alpha},
$$

so $\bar{\beta}^{2}=\bar{\alpha}^{-2}=\operatorname{Ad}\left(f-f^{\prime}\right)$. Moreover,

$$
\begin{aligned}
\bar{\beta}^{-1} \bar{\alpha}=\bar{\alpha}(\bar{\beta} \bar{\alpha})^{-1} \bar{\alpha} & =\bar{\alpha} \operatorname{Ad}\left(f+u^{*} f^{\prime}\right) \bar{\alpha}^{-1} \bar{\alpha}^{2} \\
& =\operatorname{Ad}\left(\bar{\alpha}\left(f+u^{*} f^{\prime}\right)\right) \operatorname{Ad}\left(f-f^{\prime}\right) \\
& =\operatorname{Ad}\left(f+u f^{\prime}\right) \operatorname{Ad}\left(f-f^{\prime}\right) \\
& =\operatorname{Ad}\left(f-u f^{\prime}\right) .
\end{aligned}
$$

Hence

$$
\begin{aligned}
\bar{\beta} \bar{\alpha}+\bar{\beta}^{-1} \bar{\alpha} & =\operatorname{Ad}\left(f+u f^{\prime}\right)+\operatorname{Ad}\left(f-u f^{\prime}\right) \\
& =\operatorname{Ad}\left(f-f^{\prime}\right)+1 \\
& =\bar{\alpha}^{2}+1,
\end{aligned}
$$

so $\bar{\beta}+\bar{\beta}^{-1}=\bar{\alpha}+\bar{\alpha}^{-1}$. It follows that

$$
\pi\left(\beta(a)+\beta^{-1}(a)-\alpha(a)-\alpha^{-1}(a)\right)=0 \quad(a \in A) .
$$

But

$$
\begin{aligned}
& \beta(a)+\beta^{-1}(a)-\alpha(a)-\alpha^{-1}(a)=(\beta-\alpha)(a)-\alpha^{-1}(\beta-\alpha) \beta^{-1}(a) \in I_{1}, \\
& \beta(a)+\beta^{-1}(a)-\alpha(a)-\alpha^{-1}(a)=\left(\beta-\alpha^{-1}\right)(a)-\beta^{-1}\left(\beta-\alpha^{-1}\right) \alpha(a) \in I_{2} .
\end{aligned}
$$

Since $\pi$ is faithful on $I_{1} \cap I_{2}$, it follows that

$$
\beta(a)+\beta^{-1}(a)=\alpha(a)+\alpha^{-1}(a) .
$$

COROLLARY 3.2. Let $\alpha$ and $\beta$ be *-automorphisms of a $C^{*}$-algebra $A$ such that $\alpha+\alpha^{-1}=\beta+\beta^{-1}$. There exist ideals $I_{1}, I_{2}$ and $I_{3}$ of $A$, each invariant under $\alpha, \alpha^{-1}, \beta$ and $\beta^{-1}$, such that $I_{1} \cap I_{2} \cap I_{3}=\{0\}, I_{3} \subseteq I_{1}+I_{2}$ and, for each $a$ in $A$,

$$
\begin{array}{ll}
\beta(a)-\alpha(a) \in I_{1}, & \beta(a)-\alpha^{-1}(a) \in I_{2}, \\
\beta^{2}(a)-\alpha^{2}(a) \in I_{3}, & \beta^{2}(a)-\alpha^{-2}(a) \in I_{3} .
\end{array}
$$

ProOF. Let $I_{1}, I_{2}$, etc. be as in Theorem 3.1. Let $I_{3}$ be the smallest ideal of $A$ which contains the union of the ranges of $\beta^{2}-\alpha^{2}$ and $\beta^{2}-\alpha^{-2}$ and which is invariant under $\alpha, \alpha^{-1}, \beta$ and $\beta^{-1}$. (In fact, simple algebraic calculations show that the ideal generated by the ranges is already invariant.) Since

$$
\begin{aligned}
\beta^{2}-\alpha^{2} & =\beta\left(\beta+\beta^{-1}\right)-\alpha\left(\alpha+\alpha^{-1}\right)=(\beta-\alpha)\left(\alpha+\alpha^{-1}\right), \\
\beta^{2}-\alpha^{-2} & =\beta\left(\beta+\beta^{-1}\right)-\alpha^{-1}\left(\alpha+\alpha^{-1}\right)=\left(\beta-\alpha^{-1}\right)\left(\alpha+\alpha^{-1}\right),
\end{aligned}
$$

it is clear that $I_{3} \subseteq I_{1}+I_{2}$.

Since $\bar{\beta}^{2}=\bar{\alpha}^{2}=\bar{\alpha}^{-2}$, the ranges of $\beta^{2}-\alpha^{2}$ and $\beta^{2}-\alpha^{-2}$ are contained in the kernel of $\pi$, which is invariant, so $\pi \mid I_{3}=0$. Since $\pi$ is faithful on $I_{1} \cap I_{2}$, $I_{1} \cap I_{2} \cap I_{3}=\{0\}$. 
COROLLARY 3.3. Let $\alpha$ and $\beta$ be *-automorphisms of a simple $C^{*}$-algebra $A$, such that $\alpha+\alpha^{-1}=\beta+\beta^{-1}$. Then at least one of the following conditions holds:

(11.1) $\beta=\alpha$,

(11.2) $\beta=\alpha^{-1}$,

(11.3) $\beta^{2}=\alpha^{2}=\alpha^{-2}$, and there is a (faithful) representation $\pi$ of $A$ in which $\alpha^{2}$ is weakly inner, implemented by a symmetry in $\pi(A)^{\prime \prime}$. If $\pm i$ do not belong to the spectrum of $\alpha$, then (11.1) or (11.2) holds.

Proof. Since $A$ is simple, either $I_{1}=\{0\}$, or $I_{2}=\{0\}$, or $I_{1}=I_{2}=A$ and $\pi$ is faithful (in the notation of Theorem 3.1). The respective possibilities lead to the conclusions (11.1), (11.2) and (11.3).

Suppose that $\beta^{2}=\alpha^{2}=\alpha^{-2}$ and $\pm i \notin \sigma(\alpha)$. Since $\beta\left(1+\alpha^{-2}\right)=\beta\left(1+\beta^{-2}\right)=$ $\alpha\left(1+\alpha^{-2}\right)=\alpha^{-1}\left(1+\alpha^{2}\right)=\alpha^{-1}\left(1+\alpha^{-2}\right)$ and $1+\alpha^{-2}$ is invertible, it follows that $\beta=\alpha=\alpha^{-1}$.

COROLLARY 3.4. Let $\alpha$ and $\beta$ be *-automorphisms of a $C^{*}$-algebra $A$, such that $\alpha+\alpha^{-1}=\beta+\beta^{-1}$, and suppose that there is no pure state $\varphi$ of $A$ such that $\varphi \circ \beta^{2}=\varphi \circ \alpha^{2}=\varphi \circ \alpha^{-2}$. Then $A=A_{1} \oplus A_{2}$, where $A_{1}$ and $A_{2}$ are $C^{*}$ subalgebras of $A$, invariant under $\alpha$ and $\beta, \beta\left|A_{1}=\alpha^{-1}\right| A_{1}$ and $\beta\left|A_{2}=\alpha\right| A_{2}$.

ProOF. Suppose that, in the notation of Corollary $3.2, I_{3} \neq A$. Then there is a pure state $\varphi$ annihilating $I_{3}$, and therefore satisfying: $\varphi \circ \beta^{2}=\varphi \circ \alpha^{2}=\varphi \circ \alpha^{-2}$. Thus the assumption implies that $I_{3}=A$.

Since $I_{1} \cap I_{2} \cap I_{3}=\{0\}$ and $I_{3} \subseteq I_{1}+I_{2}$, it follows that $A=I_{1} \oplus I_{2}$. For $a$ in $I_{1},\left(\beta-\alpha^{-1}\right)(a) \in I_{1} \cap I_{2}=\{0\}$, so $\beta\left|I_{1}=\alpha^{-1}\right| I_{1}$. Similarly, $\beta\left|I_{2}=\alpha\right| I_{2}$.

COROLlARY 3.5. Let $\alpha$ and $\beta$ be *-automorphisms of a von Neumann algebra $\mathscr{K}$ such that $\alpha+\alpha^{-1}=\beta+\beta^{-1}$. Then $\mathscr{K}=\mathscr{M}_{1} \oplus \mathscr{M}_{2} \oplus \mathscr{M}_{3}$, where $\mathscr{M}_{1}, \mathscr{M}_{2}$, and $\mathscr{M}_{3}$ are von Neumann subalgebras of $\mathscr{M}$, invariant under $\alpha$ and $\beta$, $\beta\left|\mathscr{M}_{1}=\alpha\right| \mathscr{K}_{1}, \beta\left|\mathscr{M}_{2}=\alpha^{-1}\right| \mathscr{K}_{2}, \beta^{2}\left|\mathscr{M}_{3}=\alpha^{2}\right| \mathscr{K}_{3}=\alpha^{-2} \mid \mathscr{K}_{3}$.

PROOF. In the notation of Corollary 3.2, $I_{1} I_{2} I_{3} \subseteq I_{1} \cap I_{2} \cap I_{3}=\{0\}$, so that $J_{1} J_{2} J_{3}=\{0\}$, where $J_{j}$ is the ultraweak closure of $I_{j}$ in $\mathscr{M}$. There is a central projection $p_{j}$ in $\mathscr{M}$ such that $J_{j}=\mathscr{M} p_{j}, \alpha\left(p_{j}\right)=\beta\left(p_{j}\right)=p_{j}$. Then $p_{1} p_{2} p_{3}=0$. Let $\mathscr{M}_{1}=\mathscr{M}\left(1-p_{1}\right), \mathscr{M}_{2}=\mathscr{M} p_{1}\left(1-p_{2}\right), \mathscr{M}_{3}=\mathscr{M} p_{1} p_{2}=\mathscr{M} p_{1} p_{2}\left(1-p_{3}\right)$. For $a$ in $\mathscr{M}_{1}, \beta(a)=\beta\left(a\left(1-p_{1}\right)\right)=\beta(a)\left(1-p_{1}\right)=\alpha(a)\left(1-p_{1}\right)=\alpha(a)$. Similarly, $\beta\left|\mathscr{K}_{2}=\alpha^{-1}\right| \mathscr{K}_{2}, \beta^{2}\left|\mathscr{K}_{3}=\alpha^{2}\right| \mathscr{K}_{3}=\alpha^{-2} \mid \mathscr{K}_{3}$.

It is straightforward to recover Corollary 3.2 from Corollary 3.5, by putting $\mathscr{M}=A^{* *}, I_{1}=A \cap\left(\mathscr{M}_{2} \oplus \mathscr{H}_{3}\right), I_{2}=A \cap\left(\mathscr{M}_{1} \oplus \mathscr{M}_{3}\right), I_{3}=A \cap\left(\mathscr{M}_{1} \oplus \mathscr{M}_{2}\right)$. Similarly in the case when $\alpha$ and $\beta$ commute, it is possible to deduce the following result 
from [6], but it is instructive to see how the special case fits into the present strategy.

PROPOSITION 3.6. Let $\alpha$ and $\beta$ be *-automorphisms of a $C^{*}$-algebra $A$ such that $\alpha+\alpha^{-1}=\beta+\beta^{-1}$. The following are equivalent:

(i) $\alpha \beta=\beta \alpha$,

(ii) For each irreducible representation $\pi$ of $A$, either $\pi \beta=\pi \alpha$ or $\pi \beta=\pi \alpha^{-1}$,

(iii) For $a, b, c$ in $A$,

$$
(\beta(a)-\alpha(a)) b\left(\beta(c)-\alpha^{-1}(c)\right)=0,
$$

(iv) There exist ideals $I_{1}$ and $I_{2}$ of $A$ such that $I_{1} \cap I_{2}=\{0\}$ and, for each $a$ in $A, \beta(a)-\alpha(a) \in I_{1}, \beta(a)-\alpha^{-1}(a) \in I_{2}$,

(v) The ranges of $\beta-\alpha$ and $\beta-\alpha^{-1}$ have trivial intersection.

PROOF. (i) $\Rightarrow$ (ii). Let $e$ be the support of $\pi$. In the proof of Theorem 3.1, it was shown that $e \in \mathscr{E}_{1} \cup \mathscr{E}_{2}$ except possibly in case 4 when (7.3) applies. In this exceptional case, the condition (i) implies that $f_{e}=0$ or $f_{e}=e$ or $\lambda_{e}= \pm 1$. But then $e \in \mathscr{E}_{1} \cup \mathscr{E}_{2}$.

Thus, in all cases, $e \in \mathscr{E}_{1} \cup \mathscr{E}_{2}$. If $e \in \mathscr{E}_{1}$, then $\alpha^{-1}(e)=\beta^{-1}(e)$ and $\beta\left|\mathscr{M} \beta^{-1}(e)=\alpha\right| \mathscr{M} \alpha^{-1}(e)$, so $\pi(\beta(a))=\pi\left(\beta\left(a \beta^{-1}(e)\right)\right)=\pi\left(\alpha\left(a \alpha^{-1}(e)\right)\right)=$ $\pi(\alpha(a))$, so $\pi \beta=\pi \alpha$. Similarly, if $e \in \mathscr{E}_{2}$, then $\pi \beta=\pi \alpha^{-1}$.

(ii) $\Rightarrow$ (iii). Condition (ii) implies that

$$
\pi\left((\beta(a)-\alpha(a)) b\left(\beta(c)-\alpha^{-1}(c)\right)\right)=0
$$

for each irreducible representation $\pi$.

(iii) $\Rightarrow$ (iv). Condition (iii) implies that the ideals generated by the ranges of $\beta-\alpha$ and $\beta-\alpha^{-1}$ are orthogonal.

(iv) $\Rightarrow(\mathrm{v})$. This is trivial.

(v) $\Rightarrow$ (i). It follows from (1) that

$$
\begin{aligned}
& (\alpha \beta-\beta \alpha)-(\beta-\alpha)\left(\alpha^{-1}-\beta\right)=\beta\left(\beta+\beta^{-1}-\alpha-\alpha^{-1}\right)=0 \\
& (\alpha \beta-\beta \alpha)-\left(\beta-\alpha^{-1}\right)(\beta-\alpha)=\left(\alpha+\alpha^{-1}-\beta-\beta^{-1}\right) \alpha=0 .
\end{aligned}
$$

Thus the range of $\alpha \beta-\beta \alpha$ is contained in the intersection of the ranges of $\beta-\alpha$ and $\beta-\alpha^{-1}$, so condition (v) implies that $\alpha \beta=\beta \alpha$.

\section{One-parameter groups}

Let $\alpha$ and $\beta$ be actions of $\mathbf{R}$ on a $C^{*}$-algebra $A$, such that

$$
\alpha_{t}+\alpha_{-t}=\beta_{t}+\beta_{-t} \text { for all } t .
$$


For fixed $t$, Theorem 3.1 can be applied, but the results are comparatively crude. It was shown in [7] that if $A$ is a von Neumann algebra and $\alpha$ and $\beta$ are weakly continuous (that is, $\alpha_{t}(a) \rightarrow a, \beta_{t}(a) \rightarrow a$ ultraweakly as $t \rightarrow 0$, for each $a$ in $A$ ), then there is a central projection $p$ in $A$, independent of $t$ and invariant under $\alpha$ and $\beta$, such that $\beta_{t}\left|A p=\alpha_{t}\right| A p, \beta_{t}\left|A(1-p)=\alpha_{-t}\right| A(1-p)$ for all $t$.

Now suppose that $A$ is a $C^{*}$-algebra, and $\alpha$ and $\beta$ are strongly continuous $\left(\left\|\alpha_{t}(a)-a\right\| \rightarrow 0,\left\|\beta_{t}(a)-a\right\| \rightarrow 0\right.$ as $\left.t \rightarrow 0\right)$ and satisfy (2). It is not possible to apply the result for von Neumann algebras to the extensions of $\alpha$ and $\beta$ to actions on $A^{* *}$, since these extended actions may not be weakly continuous. Nevertheless, it is possible to carry through most of the steps of the proof in [7] (with appropriate interpretations of spectral subspace). Proposition 2.8 of [7] is no longer valid, but a new argument may be given to show that the norm-closure of the ideal $K_{0}$ defined on page 270 is an ideal. This then leads to a proof of Theorem 4.1 below.

Rather than giving the details of this, it seems preferable to show how the theorem for one-parameter groups may be proved by the method used for single automorphisms. The strategy is very simple - the exceptional case 4 in the proof of Theorem 3.1 is avoided by replacing $t$ by $t / 2$. One then has to establish that the ideals $I_{1}$ and $I_{2}$ can be taken to be independent of $t$, and complications arise from the absence of continuity on $A^{* *}$. Indeed, the first part of the proof is taken up by showing that the actions $\alpha$ and $\beta$ commute, from which point various relatively elementary proofs are known (see the introduction of [7]).

THEOREM 4.1. Let $\alpha$ and $\beta$ be strongly continuous one-parameter groups of *-automorphisms of a $C^{*}$-algebra $A$ such that $\alpha_{t}+\alpha_{-t}=\beta_{t}+\beta_{-t}$ for all $t$. There exist ideals $I_{1}$ and $I_{2}$, invariant under $\alpha$ and $\beta$, such that $I_{1} \cap I_{2}=\{0\}$ and, for all $a$ in $A$ and all real $t, \beta_{t}(a)-\alpha_{t}(a) \in I_{1}$ and $\beta_{t}(a)-\alpha_{-t}(a) \in I_{2}$. Moreover, $\alpha_{s} \beta_{t}=\beta_{t} \alpha_{s}$ for all real $s$ and $t$.

Proof. As in Theorem 3.1, consider the set $\mathscr{E}$ of all minimal central projections in the atomic part $\mathscr{K}$ of $A^{* *}$. For fixed real $t \neq 0$, let

$$
\begin{aligned}
& \mathscr{E}_{1}(t)=\left\{e \in \mathscr{E}: \beta_{t}\left|\mathscr{M} e=\alpha_{t}\right| \mathscr{K} e\right\}, \\
& \mathscr{E}_{2}(t)=\left\{e \in \mathscr{E}: \beta_{t}\left|\mathscr{M} e=\alpha_{-t}\right| \mathscr{M} e\right\} .
\end{aligned}
$$

By (8.2), for $e$ in $\mathscr{E}_{j}(t), \alpha_{ \pm t}(e), \beta_{ \pm t}(e) \in \mathscr{E}_{j}$, and hence, by iteration, $\mathscr{E}_{j}(t) \subseteq$ $\mathscr{E}_{j}(k t)$ for any integer $k$.

For each $e$ in $\mathscr{E}$, it is claimed that $e \in \mathscr{E}_{1}(t) \cup \mathscr{E}_{2}(t)$. As in Theorem 3.1, there are four cases, and the only one in which the claim is not immediate is Case 4.

So, suppose that $\alpha_{t}(e)=\beta_{t}(e)=\alpha_{-t}(e)=\beta_{-t}(e) \neq e$. Then $\alpha_{t / 2}(e) \neq$ $\alpha_{-t / 2}(e)$, so for the pair $\left(\alpha_{t / 2}, \beta_{t / 2}\right)$ of automorphisms, Case 4 does not apply 
to $e$. Thus $e \in \mathscr{E}_{1}(t / 2) \cup \mathscr{E}_{2}(t / 2) \subseteq \mathscr{E}_{1}(t) \cup \mathscr{E}_{2}(t)$. The claim is established, and it follows easily that $\alpha_{t} \beta_{t}=\beta_{t} \alpha_{t}$ (see Proposition 3.6).

For any integer $n$, let $t_{n}=2^{-n} t$. Replacing $t$ by $t_{n}$, it follows that $\alpha_{t_{n}} \beta_{t_{n}}=$ $\beta_{t_{n}} \alpha_{t_{n}}$ and hence $\alpha_{k t_{n}} \beta_{t}=\beta_{t} \alpha_{k t_{n}}$ for any integer $k$. Since $\left\{k t_{n}: k, n \in \mathbf{Z}\right\}$ is dense in $\mathbf{R}$, it follows from the strong continuity of $\alpha$ that $\alpha_{s} \beta_{t}=\beta_{t} \alpha_{s}$ for all real $s$ (and $t$ ).

Now, let $t$ vary, and put

$$
\begin{aligned}
& \mathscr{E}_{1}=\left\{e \in \mathscr{E}: \beta_{t}\left|\mathscr{M} e=\alpha_{t}\right| \mathscr{M} e \text { for all } t\right\}=\bigcap_{t} \mathscr{E}_{1}(t), \\
& \mathscr{E}_{2}=\bigcap_{t} \mathscr{E}_{2}(t) .
\end{aligned}
$$

For each $e$ in $\mathscr{E}$, it is claimed that

(12.1) $e \in \mathscr{E}_{1} \cup \mathscr{E}_{2}$,

(12.2) $e \in \mathscr{E}_{j} \Rightarrow \alpha_{s}(e), \beta_{s}(e) \in \mathscr{E}_{j}$ for all $s(j=1,2)$.

There are now three cases:

1. For some $t_{0}, \alpha_{t_{0}}(e) \neq \beta_{-t_{0}}(e)$,

2. For some $t_{0}, \alpha_{t_{0}}(e) \neq \beta_{t_{0}}(e)$,

3. For all $t, \alpha_{t}(e)=\beta_{t}(e)=\alpha_{-t}(e)=\beta_{-t}(e)$.

Case 1. Let $t_{n}=2^{-n} t_{0}$. Suppose that $\alpha_{t_{1}}(e)=\beta_{-t_{1}}(e)$. If $\beta_{-t_{1}}(e)=\beta_{t_{1}}(e)$, then $\beta_{t_{0}}(e)=e=\beta_{-t_{0}}(e)$, so $\alpha_{t_{0}}(e)=\alpha_{-t_{0}}(e)=e=\beta_{-t_{0}}(e)$, a contradiction. If $\beta_{-t_{1}}(e) \neq \beta_{t_{1}}(e)$, then Case 2 of the proof of Theorem 3.1 applies to $e$ for the pair $\left(\alpha_{t_{1}}, \beta_{t_{1}}\right)$, so $e \in \mathscr{E}_{2}\left(t_{1}\right) \subseteq \mathscr{E}_{2}\left(t_{0}\right)$. In particular, $\alpha_{t_{0}}(e)=\beta_{-t_{0}}(e)$, a contradiction.

Thus it follows that $\alpha_{t_{1}}(e) \neq \beta_{-t_{1}}(e)$, and hence by iteration that $\alpha_{t_{n}}(e) \neq$ $\beta_{-t_{n}}(e)$ for $n \geq 0$. Thus Case 1 of the proof of Theorem 3.1 applies to $e$ for the pair $\left(\alpha_{t_{n}}, \beta_{t_{n}}\right)$, so $e \in \mathscr{E}_{1}\left(t_{n}\right)$. For any integer $k, \alpha_{-k t_{n}}(e) \in \mathscr{E}_{1}\left(k t_{n}\right)$, so

$$
\beta_{k t_{n}}(a) e=\alpha_{k t_{n}}(a) e \quad(a \in A) .
$$

By the strong continuity in $t$,

$$
\beta_{t}(a) e=\alpha_{t}(a) e \quad(a \in A, t \in \mathbf{R}) .
$$

By the ultraweak continuity in $a$,

$$
\beta_{t}(a) e=\alpha_{t}(a) e \quad(a \in \mathscr{M}, t \in \mathbf{R}) .
$$

In particular,

$$
\beta_{t}\left(\alpha_{-t}(e)\right) e=e .
$$

Replacing $t$ by $-t$, it follows that

$$
\beta_{t}(e)=\alpha_{t}(e) \text { for all } t .
$$


Now, replacing $a$ by $\alpha_{-s-t}(a)$ and applying $\alpha_{s+t}$ to both sides of (13), using (14) and the commutativity of $\alpha$ and $\beta$,

$$
\beta_{t}\left(a \alpha_{s}(e)\right)=\alpha_{s+t}\left(\beta_{t}\left(\alpha_{-s-t}(a)\right) e\right)=\alpha_{s+t}\left(\alpha_{t} \alpha_{-s-t}(a) e\right)=\alpha_{t}\left(a \alpha_{s}(e)\right) .
$$

Thus $\alpha_{s}(e) \in \mathscr{E}_{1}$ for all $s$. Moreover, $e \notin \mathscr{E}_{2}$.

Case 2. This is similar to Case $1-$ now, $\alpha_{s}(e) \in \mathscr{E}_{2}$ for all $s, e \notin \mathscr{E}_{1}$.

Case 3. Replacing $t$ by $t / 2$, it follows that $\alpha_{t}(e)=\beta_{t}(e)=e$ for all $t$. By Case 3 of the proof of Theorem 3.1, for each $t$, either $\beta_{t}\left|\mathscr{M} e=\alpha_{t}\right| \mathscr{M} e$ or $\beta_{t}\left|\mathscr{M} e=\alpha_{-t}\right| \mathscr{M} e$.

Suppose that $e \notin \mathscr{E}_{2}$, so for some $t_{0}, \beta_{t_{0}}\left|\mathscr{M} e \neq \alpha_{-t_{0}}\right| \mathscr{M} e$. Let $t_{n}=2^{-n} t_{0}$ $(n \geq 0)$. Then $\beta_{t_{n}}\left|\mathscr{K} e \neq \alpha_{-t_{n}}\right| \mathscr{M} e$ (taking $n$th powers), so $\beta_{t_{n}}\left|\mathscr{M} e=\alpha_{t_{n}}\right| \mathscr{K} e$. Hence $\beta_{k t_{n}}\left|\mathscr{K}_{e}=\alpha_{k t_{n}}\right| \mathscr{M} e$ for all integers $k$, so

$$
\beta_{k t_{n}}(a) e=\alpha_{k t_{n}}(a) e \quad(a \in A) .
$$

By the strong continuity in $t$, and ultraweak continuity in $a$,

$$
\beta_{t}(a e)=\beta_{t}(a) e=\alpha_{t}(a) e=\alpha_{t}(a e) \quad(a \in \mathscr{M}, t \in \mathbf{R})
$$

so $e \in \mathscr{E}_{1}$. In this case, (12.2) is trivial.

The proof is now completed as in Theorem 3.1, taking

$$
I_{j}=\left\{a \in A: a e=0 \text { for all } e \text { in } \mathscr{E}_{j}\right\} .
$$

COROLLARY 4.2. Let $\alpha$ and $\beta$ be strongly continuous one-parameter groups of *-automorphisms of a simple $C^{*}$-algebra $A$ such that $\alpha_{t}+\alpha_{-t}=\beta_{t}+\beta_{-t}$ for all $t$. Then $\beta=\alpha$ or $\beta=\alpha^{-1}$.

COROLLARY 4.3. Let $\alpha$ and $\beta$ be strongly continuous one-parameter groups of *-automorphisms of a $C^{*}$-algebra $A$ such that $\alpha_{t}+\alpha_{-t}=\beta_{t}+\beta_{-t}$ for all $t$, and suppose that there is no pure state of $A$ which is invariant under both $\alpha$ and $\beta$. Then $A=A_{1} \oplus A_{2}$, where $A_{1}$ and $A_{2}$ are $C^{*}$-subalgebras of $A$, invariant under $\alpha$ and $\beta$, such that $\beta_{t}\left|A_{1}=\alpha_{-t}\right| A_{1}, \beta_{t}\left|A_{2}=\alpha_{t}\right| A_{2}$ for all $t$.

Proof. Suppose that $I_{1}+I_{2} \neq A$. Then there is a pure state $\varphi$ annihilating $I_{1}$ and $I_{2}$, so $\varphi \circ \beta_{t}=\varphi \circ \alpha_{t}=\varphi \circ \alpha_{-t}$. Hence $\varphi \circ \alpha_{2 t}=\varphi$ for all $t$, so $\varphi \circ \beta_{t}=\varphi \circ \alpha_{t}=\varphi$ for all $t$, a contradiction. Thus $A=I_{1} \oplus I_{2}$, and $\beta_{t}\left|I_{1}=\alpha_{-t}\right| I_{1}$, etc.

It is not entirely straightforward to recover the result of [7] for von Neumann algebras $\mathscr{M}$ from Theorem 4.1. If the weakly continuous actions $\alpha$ and $\beta$ on $\mathscr{M}$ are assumed (or can be shown) to commute, then there is an ultraweakly dense invariant $C^{*}$-subalgebra $A$ of $\mathscr{A}$ on which the actions are strongly continuous. 
An application of Theorem 4.1 then provides orthogonal ideals $I_{1}$ and $I_{2}$ of $A$. The identity $p$ of the ultraweak closure of $I_{2}$ in $\mathscr{M}$ then satisfies $\beta_{t}\left|\mathscr{M} p=\alpha_{t}\right| \mathscr{M} p$, $\beta_{t}\left|\mathscr{M}(1-p)=\alpha_{-t}\right| \mathscr{M}(1-p)$.

The first part of the proof of Theorem 4.1 needs only very slight modification to show the commutativity of weakly continuous actions satisfying (2).

In Theorem 4.1, it is always possible to arrange that $I_{1}+I_{2}$ is essential (by taking $I_{2}$ to be the orthogonal complement of $I_{1}$ ), but it may not be possible to arrange that $A=I_{1} \oplus I_{2}$, as the following example shows. Note, however, that, given $\alpha, I_{1}$ and $I_{2}, \beta$ is uniquely determined by the conditions: $\beta_{t}(a)-\alpha_{t}(a) \in I_{1}$, $\beta_{t}(a)-\alpha_{-t}(a) \in I_{2}$.

EXAMPLE 4.4. Let $\Omega$ be the one-point compactification of $\mathbf{R} \times\{1,2\}, A=$ $C(\Omega)$ and

$$
\begin{array}{llll}
\left(\alpha_{t} f\right)(s, 1)=f(s+t, 1), & \left(\alpha_{t} f\right)(s, 2)=f(s+t, 2), & & \left(\alpha_{t} f\right)(\infty)=f(\infty), \\
\left(\beta_{t} f\right)(s, 1)=f(s+t, 1), & \left(\beta_{t} f\right)(s, 2)=f(s-t, 2), & & \left(\beta_{t} f\right)(\infty)=f(\infty) .
\end{array}
$$

Then $\alpha$ and $\beta$ satisfy (2), and

$$
\begin{aligned}
& I_{1}=\{f \in A: f(s, 1)=0 \text { for all } s\}, \\
& I_{2}=\{f \in A: f(s, 2)=0 \text { for all } s\} .
\end{aligned}
$$

It is possible to give an infinitesimal version of Theorem 4.1, analogous to Theorem 3.1 of $[7]$.

Proposition 4.5. Let $\delta_{1}$ and $\delta_{2}$ be derivations of a $C^{*}$-algebra $A$ which are generators of strongly continuous one-parameter groups of $*$-automorphisms, and suppose that $\delta_{1}^{2}=\delta_{2}^{2}$. Then $\mathscr{D}\left(\delta_{1}\right)=\mathscr{D}\left(\delta_{2}\right), \delta_{1} \delta_{2}=\delta_{2} \delta_{1}$ and there exist ideals $I_{1}$ and $I_{2}$ in $A$ such that $I_{1} \cap I_{2}=\{0\}, \delta_{i}\left(I_{j} \cap \mathscr{D}\right) \subseteq I_{j}(i, j=1,2)$, $\left(\delta_{1}-\delta_{2}\right)(\mathscr{D}) \subseteq I_{1},\left(\delta_{1}+\delta_{2}\right)(\mathscr{D}) \subseteq I_{2}$, where $\mathscr{D}=\mathscr{D}\left(\delta_{1}\right)=\mathscr{D}\left(\delta_{2}\right)$.

PROOF. Let $\alpha$ and $\beta$ be the groups generated by $\delta_{1}$ and $\delta_{2}$, respectively. Then $\alpha_{t}+\alpha_{-t}=\beta_{t}+\beta_{-t}$ (see [7]). Let $I_{1}$ and $I_{2}$ be the ideals given by Theorem 4.1. Note that $\alpha$ and $\beta$ commute.

Suppose that $a \in \mathscr{D}\left(\delta_{1}\right)$, and let

$$
b=\int_{0}^{1} \beta_{t}(a) d t \in \mathscr{D}\left(\delta_{1}\right) \cap \mathscr{D}\left(\delta_{2}\right)
$$


Then

$$
\begin{aligned}
b-a= & \int_{0}^{1}\left(\beta_{t}(a)-a\right) d t \\
= & \int_{0}^{1} \beta_{t / 2}\left[\beta_{t / 2}(a)-\alpha_{t / 2}(a)\right] d t \\
& +\int_{0}^{1} \beta_{t / 2}\left[\alpha_{t / 2}(a)-\beta_{-t / 2}(a)\right] d t \\
\in & \mathscr{D}\left(\delta_{1}\right) \cap I_{1}+\mathscr{D}\left(\delta_{1}\right) \cap I_{2} .
\end{aligned}
$$

Since $\beta_{t}\left|I_{1}=\alpha_{-t}\right| I_{1}$ and $\beta_{t}\left|I_{2}=\alpha_{t}\right| I_{2}, \mathscr{D}\left(\delta_{1}\right) \cap I_{j}=\mathscr{D}\left(\delta_{2}\right) \cap I_{j}$. Thus $b-a \in$ $\mathscr{D}\left(\delta_{2}\right)$, so $a \in \mathscr{D}\left(\delta_{2}\right)$.

Similarly, $\mathscr{D}\left(\delta_{2}\right) \subseteq \mathscr{D}\left(\delta_{1}\right)$. The remaining parts of the Proposition are now clear.

Proposition 4.5 may fail for closed derivations which are not generators. There are numerous examples with $\mathscr{D}\left(\delta_{1}^{2}\right)=\mathscr{D}\left(\delta_{2}^{2}\right)=\{0\}$, for example.

\section{References}

[1] M. Awami and A. B. Thaheem, 'A short proof of a decomposition theorem of a von Neumann algebra', Proc. Amer. Math. Soc. 92 (1984), 81-82.

[2] M. Awami and A. B. Thabeem, 'A short proof of a decomposition theorem of a von Neumann algebra', Boll. Un. Mat. Ital. A 2 (1983), 239-240.

[3] U. Haagerup and F. Hanche-Olsen, 'Tomita-Takesaki theory for Jordan algebras', $J$. Operator Theory 11 (1984), 343-364.

[4] U. Haagerup and C. Skau, 'Geometric aspects of the Tomita-Takesaki theory II', Math. Scand. 48 (1981), 241-252.

[5] A. B. Thaheem, 'Decomposition of a von Neumann algebra relative to a *-automorphism', Proc. Edinburgh Math. Soc. 22 (1979), 9-10.

[6] A. B. Thaheem, 'Decomposition of a von Neumann algebra', Rend. Sem. Mat. Univ. Padova 65 (1981), 1-7.

[7] A. B. Thaheem, A. van Daele and L. Vanheeswijck, 'A result on two one-parameter groups of automorphisms', Math. Scand. 51 (1982), 261-274.

St. John's College

Oxford OX1 3JP

England 